Barbara Obtułowicz (D)

\title{
La vida cotidiana a bordo de un barco de vapor
}

\section{Resumen:}

La construcción en 1807 por Robert Fulton del primer barco de pasajeros y más tarde de un barco de vapor, influyó en acortar significativamente el tiempo de los viajes. Sin embargo, estos siguieron siendo largos, sobre todo en los trayectos transatlánticos. ¿Cuáles eran, entonces, las actividades de los viajeros, nuestros antepasados, a lo largo de las semanas de los cruceros? ¿A qué se dedicaban los pasajeros mientras recorrían el océano? Dar respuesta a estas y otras preguntas es posible gracias a los diarios y memorias, también la cartas, redactadas a bordo de los barcos de vapor. Ni el idioma de las anotaciones ni el trayecto del crucero influían en la vida cotidiana de los pasajeros. Dado el contexto hispanista de nuestro simposio, nos centraremos en las fuentes vinculadas con las personas relacionadas con el ámbito hispanohablante.

Palabras claves: vida cotidiana; viajes; barcos de vapor

\section{Abstract:}

\section{Everyday Life on Board of a Steamboat}

The construction in 1807 of the first passenger ship, and later a steamboat, by Robert Fulton, significantly shortened a travel time. However, travelling continued to last, especially on transatlantic routes. What were the activities of the travelers, our ancestors, throughout the cruise weeks then? What did the passengers do while they toured the ocean? It is possible to answer these and other questions thanks to the diaries, memoirs, and also the letters written on board of the steamboats. Neither a language of these annotations, nor a trail of a cruise influenced the daily 
lives of the passengers. Given the Hispanic context of our symposium, we will focus on the sources related to Spanish-speaking people.

Keywords: daily life; travels; steamboats

La construcción del vapor en 1763 por James Watt es uno de los sucesos más significativos que dieron lugar al principio de la revolución industrial. Poco a poco, el invento fue aplicado a difrerntes ramas de la incipiente industria y el transporte de la época. En el cambio de los siglos XVIII y XIX una máquina de vapor era capaz de abastecer hasta cien talleres de costura. De ese modo se desarrolló la industria costurera en Inglaterra. En 1807 Robert Fulton construyó con éxito el primer barco de vapor para el uso de pasajeros y luego otro tipo de embarcación propulsada por vapor. En 1819 el buque de vapor "Savannah" recorrió el Atlántico por primera vez (de Nueva York a Liverpool). El ingeniero británico, George Stephenson, diseñó la moderna versión de locomotora de vapor y, por consiguiente, se inauguró el primer ferrocarril en el tramo Liverpool-Manchester (1830). El rápido progreso y desarrollo de los nuevos medios de comunicación, y la modernización de los antiguos, influyeron en la reducción del tiempo de los viajes. A pesar de eso, la duración de los recorridos aún era larga. Hoy en día, el vuelo de España a México, sin transbordos, dura alrededor de diez horas, mientras que en la segunda mitad del siglo XIX el mismo viaje a bordo de una embarcación de vapor requería un mes.

¿A qué se dedicaban entonces nuestros antepasados cuando viajaban en los barcos de vapor durante los cruceros de varias semanas? ¿Cómo amenizaban sus largas jornadas de viaje? Responder a estas preguntas es posible gracias a los diarios, las memorias y las cartas redactadas a bordo de los barcos. En la época estudiada transcribir las observaciones al papel era considerado "de buen gusto" y formaba parte de un pasatiempo casi obligatorio para la gente culta que sabía manejar la pluma. Los pasajeros que en su momento, a causa de diferentes razones, no consiguieron crear sus apuntes, después se lamentaban de no haberlo hecho. Al llegar a su destino trataban de recuperar 
los detalles relacionados con el trayecto efectuado. Pero no siempre resultaba posible, lo que pudo experimentar p.ej. el príncipe Vitoldo Adán Czartoryski que en sus múltiples periplos se dejaba vencer por la pereza ${ }^{1}$.

Hay que subrayar que ni el idioma en el que se redactaban las notas, ni el trayecto eran de gran importancia. La vida cotidiana de los pasajeros de los barcos de vapor, independientemente de su nacionalidad o el destino, era parecida. No obstante, teniendo en cuenta el carácter hispanista de nuestro simposio, nos enfocamos en las fuentes escritas por las personas relacionadas con el mundo hispanohablante. Así, para el análisis han sido elegidas dos relaciones de una extensión más grande y algunas otras que pueden ser consideradas como menores. La primera, de Mauriece Delezé Spurch, el ayudante de cámara del archiduque de México Maximiliano I, relata el viaje del emperador y su esposa Carlota Amalia de Coburgo de Trieste a Veracruz, que transcurrió entre el 14 de abril y el 28 de mayo de 1864 en la fragata de vela y vapor "Novara" (Moncada Galán, 2000: 11-66). La segunda, son los apuntes de José de Campo y Navas, el redactor jefe del diario conservador español "Correspondencia de España". En noviembre de 1873 tuvo lugar un crucero de La Habana a Puerto Rico a bordo de la embarcación de vapor “Antonio López". Le acompañaba el Ministro de Ultramar Eugenio San Jurjo, a quien le dedicó la obra² ${ }^{2}$ Las otras fuentes de la lista de relatos vinculados a España, son las cartas de los

${ }^{1}$ Vitoldo adamíta, sin falsa modestia, su descuido respecto a este tema, sobre todo lo hacía en las cartas dirigidas a su padre Adán Jorge Czartoryski, escritas entre otras ocasiones - tras el crucero de Marsella a Livorno y de París a Londres, a través del Canal de la Mancha (BCz, 7090 III, V. Czartoryski a A.J. Czartoryski, Roma, 17 XI 1846 y BCz, 6322 IV, vol. 1, escritas de camino a Aviñón, 14 II 1848).

${ }^{2}$ Archivo Histórico Nacional en Madrid (AHN), Diversos-Colecciones, 3, núm. 198, Autógrafo de José María del Campo y Navas: Periódico burlesco redactado por el Director de la "Correspondencia de España” D. José Campo y Navas durante la navegación a la Habana con el Ministro de Ultramar en 1873, en el Vapor "Antonio López". 
príncipes Czartoryski, es decir, de Ladislao, del ya mencionado Vitoldo y su esposa María Czartoryska de soltera, Grocholska ${ }^{3}$.

La principal incomodidad para los pasajeros de los buques de vapor era el omnipresente aburrimiento y la monotonía. Por eso, los viajeros buscaban distracciones más o menos útiles o gloriosas. Maurice Delezé Spruch, a parte de cumplir con el papel de ayudante de cámara, enseñaba español al emperador, y cuando tenía un rato libre, escribía sus memorias o seducía a las mujeres. Le atrajo especialmente la condesa Paola, la criada de la emperatriz Carlota. En una noche estrellada consiguió su objetivo o, más bien, terminó seducido por ella, y los dos acabaron en el camarote de esa señorita encantadora.

Y la pareja imperial, ¿cómo pasaba su tiempo libre? No seguían los pasos de Maurice ni prestaban demasiada atención el uno al otro. Por las noches dormían cada uno en su cámara y no se visitaban. Sus intereses eran incompatibles. Sobre todo, pensaban en su futuro como emperadores de México y sufrían las molestias del viaje que, como creían, luego iba a ser recompensado con la promesa del trono tan deseado. La atención de Carlota y Maximiliano se concentraba en preocupaciones secundarias de escasa importancia. No reflexionaban sobre la inseguridad política del país, que tenía que ver con la guerra civil y el enfrentamiento entre los republicanos y los monarquistas, sino que proyectaban cómo organizar "a su manera" la nueva vida en un lugar desconocido y de qué forma presentarse a sus nuevos subordinados. Meditaban sobre cómo vestirse para la ceremonia de bienvenida, primero en Veracruz, luego en la Ciudad de México. Planeaban el protocolo y las reglas de la propia ceremonia y las que iban a emplear en la corte. Pensaban en su futuro entorno personal en la

${ }^{3}$ Vitoldo Czartoryski en los años 1845-1846 había finalizado el servicio militar en Madrid en el regimiento Reina Gobernadora (Obtułowicz, 2014a: 103126). Su esposa María, junto con su hermano Tadeo Grocholski, en 1860 visitó España perfeccionando sus habilidades plásticas en varias galerías de pintura (Obtułowicz, 2014b: 69-80). Ladislao Czartoryski, mientras tanto, contrajo matrimonio con María Amparo, la hija de la reina María Cristina de Borbón, y mantenía contacto con los miembros del gobierno español y viajaba a España "para hacer negocios" (Obtułowicz, 2013: passim). 
administración y en el gobierno, y proyectaban las responsabilidades de cada uno. Redactaban sus diarios de viaje por separado y, en ellos, se pueden notar dos visiones diferentes del mismo mundo. Escribían cartas a sus familias y conocidos y se dedicaban a la lectura. Carolina leía las obras de Alejandro Humboldt, Calderón de la Barca, Emmanuel Domenech, mientras que Maximiliano se inclinaba hacia los libros históricos y militares. Ambos se quejaban de la monotonía que solo se interrumpía esporádicamente gracias a los bailes de máscaras y conciertos de música. Les molestaba el comportamiento de los miembros de la corte que no estaban acostumbrados a largos viajes y manifestaban, de una manera ostentosa, su desencanto por la morosidad del tiempo a bordo del barco. A Carlota le agobiaban las conversaciones banales, las palabras vanas, los desfiles de las damas en la cubierta de la embarcación, que tenían como único objetivo exhibir nuevas prendas y un peinado rebuscado. Maximiliano, siendo un marinero experimentado, que recorría el océano por segunda vez $z^{4}$, veía esas prácticas con distanciamiento y, junto con el cocinero Josef Tüdös y otro ayudante de cámara, Antonio Grillo, gastaba bromas. Carolina no compartía el sentido del humor de su marido. Siendo una mujer fría y distanciada, no le gustaba la compañía, se encerraba en su cámara y se enteraba de lo que ocurría en el mundillo de la embarcación a través del colaborador de confianza del emperador, Franz Kaplan Hoffinger, responsable del correo dirigido a su majestad.

A pesar de su naturaleza melancólica y carácter ameno, Maximiliano mostraba más diligencia. Se paseaba por la cubierta del barco, contemplaba el mar y miraba el horizonte a través de binoculares; fumaba habanos, conversaba con los marineros y el personal del barco; curioseaba en la cocina preguntando por el menú del día y distraía a los cocineros. En la mesa se dedicaba a largas y acaloradas conversaciones deleitándose con el vino y las tapas. Le gustaba dedicarse a la pintura y dibujar los elementos de los uniformes destinados a sus subordinados, también estudiaba español.

${ }^{4}$ Su primer viaje lo había realizado a Brasil, en 1859, para conocer la fauna y la flora del país. 
Una vez, Mauricio observó que el archiduque, al disfrutar de su tiempo libre, se dedicaba a otro tipo de prácticas. Una mañana advirtió que la puerta de su cámara estaba entreabierta y se atrevió a entrar sin llamar. Se quedó pasmado. Maximiliano, creyendo que estaba solo, se encontraba frente al espejo y ensayaba, como un actor profesional, diferentes papeles que creía que le esperaban en los primeros días, al llegar a tierra mexicana. Llevaba puesta una corona de papel, diseñada en París, que imitaba la corona real que llevaría puesta al otro lado del océano. Las poses y los gestos del monarca Habsburgo, reflejadas en el espejo, se parecían a las de un bufón. A Mauricio, por un lado, le daban ganas de reírse pero, por por otra parte, lo que vio le llevó a reflexionar sobre el futuro del II Imperio de México. Nadie sabía lo que podía pasar allí exactamente. Menos todavía se intuía que el final llegaría pronto y sería trágico para la nueva pareja imperial ${ }^{5}$.

No menos cómico era el aseo diario del archiduque que transcurría en compañía de las personas más confiadas, entre las cuales se encontraba el autor de las memorias analizadas. El colmo parecía ser el acto de peinar la larga y dorada barba del archiduque, ejercido por Grillo, y que requería, primero, repartir la barba en dos partes iguales y separarla a ambos lados. Maximiliano le daba gran importancia al aseo y consideraba este ejercicio como un acto mágico, casi sagrado y, si por alguna razón el resultado no le satisfacía, se frustraba (Moncada Galán, 2000: 18-85).

El relato de Campo y Navas se diferencia del anterior por su originalidad formal, el estilo y el humor, así como por presentar más información sobre la vida a bordo del barco. El objetivo del autor era crear una crónica particular de los acontecimientos destinada a los lectores que le eran coetáneos y a las futuras generaciones. Conforme con sus intereses y con el papel de editor de uno de los principales periódicos madrileños, sus apuntes adquirieron un cariz periodístico. Los dividió en siete números de diferentes títulos: "El Chocolate", "El Café" (dos

${ }^{5}$ Maximiliano I fue fusilado por orden de Benito Juárez en junio de 1867 y Carlota, tras fracasar en su intervención frente a Napoleón III y Pío IX, enloqueció. 
números del mismo título), "El Café pero con leche", "El Café media taza del día", "La Crónica", "El Café media taza de este día".

¿Por qué estos títulos? Porque, como señala Campo y Navas, el periódico tenía características satíricas y entre sus objetivos se encontraba el deseo de presentar información de carácter sensacionalista bien presentada. También el chocolate y el café eran productos apreciados por los españoles, que ocupaban un lugar importante en la economía del país y en los hogares. Por otro lado, eran y siguen siendo consumidos con gran gusto durante las travesías. Fijémonos entonces, si los títulos apetitosos van seguidos de un contenido parecido.

Entre los informes más emocionales predominan unos sobre el estado de salud de los pasajeros y sobre los médicos que, tras cobrar una cantidad de dinero acordada, garantizaban aliviar los dolores del trópico e incluso curar el cáncer; también sobre la caza de las ratas que al ser perseguidas saltaban al mar; y sobre los fallecimientos a bordo. Estos últimos, aunque atormentadores, presentados con una pizca de ironía, nos muestran el destino de los que perdieron sus vidas durante el viaje. Campo y Navas describe la muerte de un soldado catalán llamado Pérez que embarcó estando enfermo. Cuando la embarcación pasaba al lado de Gibraltar quiso bajar a tierra firme pero no se le permitió hacerlo. Tras su fallecimiento, el cura celebró la misa, presenciada por la mayoría de los pasajeros, se envolvió el cuerpo del muerto en una manta $y$, acto seguido, fue tirado al océano. Los pasajeros, en este momento dramático y a la vez trágico, mostraron una gran empatía y apoyaron a la familia de Pérez, que no llevaba ningún dinero consigo, además sus familiares eran pobres, y recogieron la cantidad necesaria de fondos para financiar el funeral. Campo y Navas, junto con su compañero San Jurjo pagaron 20 pesos. En total, se recaudaron 230 pesos. Un caso parecido ocurrió con una tal Dolores Cuéllar, cuando se consiguieron juntar 510 pesos.

También Causan tristeza las informaciones sobre los accidentes casuales que sufrían, sobre todo, los miembros de la tripulación al ejercer su trabajo. A uno de ellos una máquina le aplastó el dedo. Campo y Navas usó este acontecimiento para advertir que: “¡no se debe meter los dedos en cualquier sitio!” En otra ocasión, un soldado, 
apresurado por recibir su ración de comida, tropezó con un escalón, tiró la escudilla con el contenido caliente y sufrió quemaduras en la cara. "El que quiera no quemarse toma las cosas con calma, o forma su rancho aparte - para comerla a sus anchas", advertía el cronista (citas de: AHN, Campo y Navas: 28).

Otro tipo de informes que tenían la intención de servir de contrapeso a las enfermedades, muertes y accidentes, eran las noticias sobre los coqueteos y romances. Campo y Navas se quejaba de que a bordo escaseaban las mujeres. Al mismo tiempo, escribía sobre las señoras que en busca de aventuras amorosas y dinero manifestaban, sin reparo, sus deseos. Por ejemplo, una viuda empobrecida declaraba su disposición para acoger a uno o más caballeros en su camarote para pasar momentos de placer tanto de día como de noche. Añadía que era atractiva y tenía a una hija por casar. Otra jovenzuela que estaba sin compromiso decía que se había perdido a bordo y necesitaba que alguien se hiciera cargo de salvarla. A cambio ofrecía "todo lo que fuera necesario". Ocurrían también situaciones cómicas. Un "ciudadano" se paseaba por la cubierta con la cara sucia y contaba que le encantaba comer carbón vegetal. Esa extravagancia conmovió a todos los pasajeros. Empezaron a preguntarse por qué lo hacía. Algunos opinaban que era por falta de hierba a su alrededor, otros decían que se trataba de problemas intestinales, había también opiniones que intentaba llamar la atención, o simplemente que se había ensuciado la cara a propósito con polvo u hollín.

Generalmente, corrían varios rumores, ya que la gente, sin tener de que ocuparse, sufría por exceso de tiempo. Los pasajeros se miraban mutuamente y observaban lo que pasaba a su alrededor. Un punto obligatorio era seguir a diario el tiempo, observar los fenómenos atmosféricos, el horizonte, los crepúsculos, la superficie del agua y el cielo. De un gran interés gozaban los instrumentos ópticos, para poder usarlos los pasajeros hacían cola, también de noche, cuando el firmamento relucía iluminado por las estrellas, como un bordado maravilloso. Se intentaba descifrar las constelaciones y se contemplaba la vía láctea.

Aparte de todo lo anteriormente expuesto, en el vapor "Antonio López" transcurría una vida normal: se prestaba atención a los olores 
que se desprendían de la cocina adivinando lo que iba a ser servido a la hora de comer; se fumaban puros y cigarros; se escuchaba la música que tocaba la orquesta en la cubierta; se conversaba. Los pasajeros, conscientes de que compartían el mismo destino, trataban de integrarse. La mayoría de ellos, sin embargo, ya pensaba en su futuro próximo y trazaba los planes sobre qué sería de ellos al llegar a su destino. Campo y Navas se preparaba para sus nuevos deberes al anclar en Cuba y Puerto Rico. Los fragmentos de sus notas comentadas aquí fueron redactados en forma de un periódico ficticio que preparaba para ocupar su tiempo libre y también para dar testimonio a los futuros lectores. Sus apuntes él mismo los trataba también como un ejercicio para los artículos que escribiría después sobre los temas políticos y económicos. Lo indican los textos donde, con la verbosidad y el sentido del humor que le caracterizaba, interpretaba los acontecimientos en la escena política euro-atlántica (AHN, Campo y Navas: passim).

A su vez, Ladislao Czartoryski, en sus cartas dirigidas a su esposa, escritas en septiembre de 1855 a bordo del barco de vapor "Thabor", recorriendo el trayecto de Marsella a Constantinopla, presta una atención especial al propio trayecto, a las condiciones temporales y describe la naturaleza. Se nota el cuidado del aspecto plástico, en ocasiones, la presentación del contenido del texto toma una forma muy poética. Es probablemente el resultado de haberse casado unos meses antes con la española María Amparo Muñoz y de Borbón, una mujer romántica, así que su intención era satisfacer a su esposa. Además, a causa del aburrimiento del viaje, tenía tiempo suficiente para cuidar cada palabra que escribía. Él mismo decía que escribir las cartas a María Amparo y pensar en ella era la mejor tarea a la que pudo dedicarse a bordo de la embarcación. Lo único que le complicaba su quehacer eran las brisas nocturnas, el calor y el sacudimiento del barco, que le causaban malestares y problemas gástricos. Asimismo, el príncipe esbozaba el estado cambiante de la naturaleza: el cielo azul, la superficie infinita del Mediterráneo, los amaneceres y las puestas del sol. Informaba sobre su horario del día, por el cual nos enteramos de que el desayuno se servía a las 9:30, la comida a las 17:00, y los platos correspondían a un menú inglés. Las paradas del barco dependían del capitán. Cerca 
de Sicilia les seguía una bandada de tiburones y en Malta, a causa de una epidemia de cólera, tuvieron que pasar dos días de cuarentena ${ }^{6}$.

Bonitas, aunque cortas, las descripciones de los paisajes marinos tampoco le eran ajenas a Vitoldo que andaba siempre despistado. Como si quisiera recompensar a su padre por su descuido en redactar el diario de viaje, desde Roma le informaba de que el crucero, con trayecto de Marsella al puerto de Livorno, lo había pasado maravillosamente porque hacía buen tiempo y las vistas eran encantadoras: "había luna en el cielo y tiritaban las estrellas, el mar permanecía fosfórico y a lo lejos se divisaba un faro [...], alrededor de nosotros rondaban los barcos de diferente tamaño, que se distinguían por sus velas bajo el fondo oscuro del cielo"?.

También la esposa de Vitoldo sentía remordimientos por no ser capaz de dedicarle el tiempo suficiente a los apuntes de viaje. Todo eso fue a causa de su pasión por la pintura. María, en otoño de 1860, junto con su hermano Tadeo (después convertido en un pintor famoso), se fue de viaje a España. Visitaron el Norte del país, que vieron desde la cubierta del barco de vapor, pasaron unas horas en cada puerto que encontraban por el camino. Luego, por tierra, partieron hacia Valladolid, Madrid, Sevilla y Gibraltar, desde donde se dirijieron de vuelta, de nuevo por mar, a Marsella. Ya que el objetivo de su viaje era conocer la cultura pictórica de España, sobre todo las galerías de pintura, todo el tiempo libre, que pasaban tanto en mar como en tierra, lo dedicaban al dibujo, hacían esbozos y pintaban. Los caballetes los extendían en la cubierta del barco, en las galerías y museos, entre otros, en el Museo del Prado. Lo que pudo atraer su atención, aparte del arte, eran los "placenteros olores" provenientes de la cocina del barco. Les llamaban la atención de una forma muy especial, porque la cocina española les había resultado decepcionante ${ }^{8}$.

${ }^{6} \mathrm{BCz}, 7372$ III, Carta de L. Czartoryski a M.A. Czartoryska, a bordo del barco de vapor "Thabor", 14, 15, 16 y 19 IX 1855.

${ }^{7} \mathrm{BCz}, 7090$ III, Carta de L. Czartoryski a A.J. Czartoryski, Roma, 17 XI 1846.

${ }^{8} \mathrm{BCz}, 7408$ III, Carta de M. Czartoryska, de soltera Grocholska, a I. Działyńska, de soltera Czartoryska, Madrid, 2 XI, 7 XI 1860 y Gibraltar, 19 XI 1860. El viaje de María lo menciona también (Gil, 2000: 33). 
Las relaciones de los viajes a bordo de las embarcaciones de vapor en la segunda mitad del siglo XIX son un espejo en el que se refleja, por un lado, el temperamento de sus autores, y por otra parte proporcionan información sobre otros viajeros, su comportamiento en unas condiciones excepcionales. La manera en la que se pasaba el tiempo a bordo de los barcos de vapor dependía de varios factores, como la posición social, el grado de la educación, los intereses y talentos, etc. Las personas que sabían leer y escribir se dedicaban a la lectura de libros y prensa, y hacían apuntes del viaje (en forma de cartas, diarios o memorias). Su ocio era también conversar con otros viajeros, seguir los cambios de tiempo y contemplar los paisajes marinos. Se observaban unos a otros, bromeaban sobre sí mismos, pero en los momentos difíciles y dramáticos había solidaridad. Muchas personas sufrían varios malestares, la salud les fallaba, como resultado del movimiento del barco, en particular durante las tormentas o cambios climáticos. Los pasajeros se encontraban durante las comidas y las celebraciones, también en los funerales. Todos esperaban un exitoso fin de viaje y se preparaban para lo que iban a hacer al llegar a su destino.

A principios del siglo XXI la mayoría de nosotros se traslada en aviones, coches y trenes. Los barcos de vapor terminaron en los museos y solo algunos pocos pueden realizar un viaje a bordo de este medio de transporte histórico. A causa del desarrollo y progreso de las nuevas formas en las que nos comunicamos, se pierde la costumbre de plasmar las impresiones de los viajes que uno realiza. De ese modo, nos condenamos, y lo mismo ocurre con nuestras memorias, a un eterno olvido. ¡Es una pena!

\section{Bibliografía}

Manuscritos:

Biblioteca de los Príncipes Czartoryski (BCz), ms: 6322 IV, t. 1; 7090 III; 7372 III; 7408 III.

Archivo Histórico Nacional en Madrid (AHN), ms: Diversos-Colecciones, 3, nr 198, Autógrafo de José María del Campo y Navas: Periódico burlesco 
redactado por el Director de la "Correspondencia de España” D. José Campo y Navas durante la navegación a la Habana con el Ministro de Ultramar en 1873, en el Vapor "Antonio López".

Fuentes publicadas:

MONCADA GALÁN, R. (2000), Memorias de Maurice Delezé Spruch. Ayuda de cámara del emperador Maximiliano de Habsburgo (1864-1868), Publicaciones del Instituto de Cultura de Morelos, Morelos.

Estudios:

OBTUŁOWICZ, B. (2014a), "Służba wojskowa księcia Witolda Czartoryskiego w Hiszpanii (1845-1846)", Roczniki Humanistyczne, LXII, 2, pp. 103-126.

OBTUŁOWICZ, B. (2014b), "Viaje de María y Tadeo por España (1860)", en: Taracha C., de la Fuente P. (coord.), Entre Oriente y Occidente: Actas del Primer Congreso de Hispanistas, Lublin 5-7 de junio de 2013, Werset, Lublin, pp. 69-80.

OBTUŁOWICZ, B. (2013), María Amparo Muñoz y de Borbón, księżna Czartoryska, Universitas, Kraków.

GIL, C. (2000), Księżna w trepkach. Matka Maria Ksawera Czartoryska karmelitanka bosa (1833-1928), Wydawnictwo Karmelitów Bosych, Kraków. 